\title{
Automatic and Controlled Semantic Retrieval: TMS Reveals Distinct Contributions of Posterior Middle Temporal Gyrus and Angular Gyrus
}

\author{
James Davey, ${ }^{1}$ Piers L. Cornelissen, ${ }^{2}$ Hannah E. Thompson, ${ }^{1}{ }^{\mathbb{C}}$ Saurabh Sonkusare, ${ }^{1}$ Glyn Hallam, ${ }^{1}$ \\ Jonathan Smallwood, ${ }^{1}$ and ${ }^{\circ}$ Elizabeth Jefferies ${ }^{1}$ \\ ${ }^{1}$ Deparment of Psychology and York Neuroimaging Centre, University of York, York Y010 5DD, United Kingdom, and ${ }^{2}$ Department of Psychology, \\ Northumbria University, Tyne and Wear NE1 8ST, United Kingdom
}

Semantic retrieval involves both (1) automatic spreading activation between highly related concepts and (2) executive control processes that tailor this activation to suit the current context or goals. Two structures in left temporoparietal cortex, angular gyrus (AG) and posterior middle temporal gyrus (pMTG), are thought to be crucial to semantic retrieval and are often recruited together during semantic tasks; however, they show strikingly different patterns of functional connectivity at rest (coupling with the "default mode network" and "frontoparietal control system," respectively). Here, transcranial magnetic stimulation (TMS) was used to establish a causal yet dissociable role for these sites in semantic cognition in human volunteers. TMS to AG disrupted thematic judgments particularly when the link between probe and target was strong (e.g., a picture of an Alsatian with a bone), and impaired the identification of objects at a specific but not a superordinate level (for the verbal label "Alsatian" not "animal"). In contrast, TMS to pMTG disrupted thematic judgments for weak but not strong associations (e.g., a picture of an Alsatian with razor wire), and impaired identity matching for both superordinate and specific-level labels. Thus, stimulation to AG interfered with the automatic retrieval of specific concepts from the semantic store while stimulation of PMTG impaired semantic cognition when there was a requirement to flexibly shape conceptual activation in line with the task requirements. These results demonstrate that $\mathrm{AG}$ and PMTG make a dissociable contribution to automatic and controlled aspects of semantic retrieval.

Key words: angular gyrus; control; posterior middle temporal gyrus; semantic; transcranial magnetic stimulation

Significance Statement

We demonstrate a novel functional dissociation between the angular gyrus (AG) and posterior middle temporal gyrus (pMTG) in conceptual processing. These sites are often coactivated during neuroimaging studies using semantic tasks, but their individual contributions are unclear. Using transcranial magnetic stimulation and tasks designed to assess different aspects of semantics (item identity and thematic matching), we tested two alternative theoretical accounts. Neither site showed the pattern expected for a "thematic hub" (i.e., a site storing associations between concepts) since stimulation disrupted both tasks. Instead, the data indicated that PMTG contributes to the controlled retrieval of conceptual knowledge, while AG is critical for the efficient automatic retrieval of specific semantic information.

\section{Introduction}

Conceptual knowledge drives thought and behavior through a combination of (1) automatic spreading activation between highly re- lated concepts, and (2) control processes tailoring activation to suit the current context and goals (Badre et al., 2005; Whitney et al., 2012; Jefferies, 2013; Noonan et al., 2013). On encountering words and

The authors declare no competing financial interests.

This article is freely available online through the J Neurosci Author Open Choice option.

Correspondence should be addressed to Elizabeth Jefferies, Department of Psychology, University of York, York Y010 5DD, UK. E-mail: beth.jefferies@york.ac.uk.

DOI:10.1523/JNEUROSCI.4705-14.2015

Copyright $\odot 2015$ Davey et al.

This is an Open Access article distributed under the terms of the Creative Commons Attribution License Creative Commons Attribution 4.0 International, which permits unrestricted use, distribution and reproduction in any medium provided that the original work is properly attributed. 
objects, we activate representations in anterior temporal lobes (ATLs) that capture conceptual similarities extracted from perceptual/motor features (Rogers et al., 2004; Patterson et al., 2007). However, we know a myriad of features and associations for any given concept; and since only some of these are relevant at any one time, executive semantic processes are recruited to flexibly shape retrieval toward relevant but nondominant aspects (Thompson-Schill et al., 1997; Wagner et al., 2001; Jefferies and Lambon Ralph, 2006). Thus, the ATL hub interacts with control processes supported by left inferior frontal gyrus (LIFG; Thompson-Schill et al., 1997; Wagner et al., 2001; Badre et al., 2005; Noonan et al., 2013) and other sites within the frontoparietal "multiple-demand" executive system (Duncan, 2010). Greater control over conceptual retrieval is required when there is strong competition from alternative meanings and/or when the context only weakly cues the retrieval of relevant information (Badre et al., 2005; Bedny et al., 2008; Noonan et al., 2010, 2013; Whitney et al., 2011b; Rodd et al., 2012).

While ATL and LIFG are associated with automatic and controlled semantic retrieval, respectively, the contribution of temporoparietal regions remains unclear, even though posterior middle temporal gyrus (pMTG; Visser et al., 2012; Noonan et al., 2013) and mid-angular gyrus (AG; Binder et al., 2009; Humphreys and Lambon Ralph, 2014) show highly reliable responses to semantic contrasts. Some theories propose a temporoparietal semantic store complementing the information in ATL (Schwartz et al., 2011): ATL might integrate knowledge about concrete multimodal features, supporting the categorical organization of knowledge (Tyler et al., 2003; Rogers et al., 2004; e.g., apple is similar to banana), while AG and/or pMTG capture thematic knowledge (such that apple is linked to pie; Schwartz et al., 2011; Mirman and Graziano, 2012a; de Zubicaray et al., 2013).

Alternatively, pMTG and/or AG might contribute to specific conceptual retrieval processes as opposed to aspects of representation (Cabeza et al., 2011; Humphreys and Lambon Ralph, 2014). Patients with semantic aphasia (SA) following left temporoparietal stroke have deficits of controlled semantic retrieval: this "deregulated" semantic cognition is also seen following LIFG damage and is qualitatively distinct from degraded semantic knowledge seen in semantic dementia (Jefferies and Lambon Ralph, 2006; Noonan et al., 2010). However, this retrieval hypothesis remains highly controversial given that both regions are often considered to underpin aspects of semantic representation (Martin, 2007; Binder et al., 2009). Moreover, the lesions in these patients prevent the separation of pMTG and AG, which might have dissociable functions. While these two regions can show activation to semantic contrasts that is similar to that in functional neuroimaging studies (Binder et al., 2009), a recent meta-analysis (Noonan et al., 2013) showed stronger responses in pMTG as well as LIFG to demanding semantic judgments requiring control over conceptual retrieval compared with easier judgments across studies. In contrast, mid-AG typically shows deactivation relative to rest, which is greater for more difficult semantic and nonsemantic tasks (Seghier et al., 2010; Wirth et al., 2011; Humphreys and Lambon Ralph, 2014; Humphreys et al., 2015). These observations are consistent with the view that AG may support automatic aspects of retrieval, while pMTG contributes to controlled semantic retrieval.

To elucidate the different functional roles of these regions of temporoparietal cortex, we used transcranial magnetic stimulation (TMS) in healthy volunteers to produce focal "virtual lesions" in pMTG and AG, and explored the impact on identity and thematic association matching tasks. We tested the following two alternative hypotheses: (1) that AG and/or pMTG can be characterized as a "thematic hub"; and (2) that these sites make dissociable contribu- tions to automatic and controlled aspects of semantic retrieval, respectively.

\section{Materials and Methods}

Overview. We used inhibitory off-line TMS (pulses at $1 \mathrm{~Hz}$ for $10 \mathrm{~min}$ ) to produce virtual lesions within left mid-AG and PMTG, and examined the effect of this stimulation on two types of word-picture matching, requiring either (1) object identification (e.g., is the picture a Dalmation or a corgi?) or (2) the retrieval of thematic associations (e.g., does the picture of the Dalmation go with bone or feather?). Since stimulation was delivered off-line, TMS-induced disruption could not be attributed to distracting jaw contractions or eye blinks following peripheral nerve stimulation. (3) We also included a control task (scrambled picture matching) and a control site (vertex) to test for nonspecific effects of TMS. Performance immediately after the application of TMS was compared with baseline data collected either immediately before or $30 \mathrm{~min}$ after stimulation, by which time inhibitory TMS effects are no longer expected to be present (Pobric et al., 2010; Whitney et al., 2011b). This design allowed the order of TMS and baseline sessions to be counterbalanced across participants. We conducted three complementary analyses of these data. Analysis 1 provides an omnibus model comparing TMS and no-TMS trials at each site across all of the tasks. Analyses 2 and 3 examined the thematic association and identity-matching tasks, respectively, examining the data from PMTG and AG in more detail. In Analysis 2 , we assessed the effect of strength of association within the thematic matching task, treating this predictor as a continuous variable, and controlling for the effects of psycholinguistic variables and nonspecific effects of TMS. In Analysis 3, we examined the effects of specificity on identity matching while controlling for the effects of typicality and familiarity plus nonspecific effects of TMS.

Selection of stimulation sites. Figure 1 shows the stimulation sites in mid-AG and PMTG on an inflated cortical surface. These sites were taken from two complementary meta-analyses of neuroimaging data. The left mid-AG site was taken from a meta-analysis (Humphreys and Lambon Ralph, 2014) examining semantic tasks versus difficulty-matched nonsemantic tasks and is comparable to the peak for semantics reported by Binder et al. (2009). The pMTG site, in contrast, was a peak response in a meta-analysis examining diverse manipulations of semantic control (i.e., effects of strong competition, weak probe-target relationships, and impoverished contextual constraints vs lower-control versions of these tasks; Noonan et al., 2013). Thus, while the response to semantic manipulations in neuroimaging studies often encompasses both pMTG and AG within one cluster (Binder et al., 2009), we predicted that these sites would show a functional dissociation, with a greater contribution to automatic aspects of retrieval in mid-AG and more controlled semantic processes in pMTG. These temporoparietal sites are sufficiently distant to allow their separate stimulation and thus permit dissociable effects of TMS. However, we cannot rule out the possibility that some TMS was also delivered to adjacent cortical regions. In Figure 1, these locations are superimposed on maps of resting-state connectivity, which were computed using the TMS sites as seeds. The maps were produced using resting-state fMRI data from the Enhanced Nathan Kline Institute Rockland Sample (i.e., a large publicly available set of resting-state data; Nooner et al., 2012), and reveal that our AG and pMTG sites fall within distinct networks. Stimulation of an additional site in ATL $(-53,0,-22)$ did not elicit any inhibitory effects and is not discussed further. In addition to sites implicated in semantic cognition, we applied stimulation to a control site, the vertex, in each participant. Each of these sites was stimulated for each participant in a different session; participants had two sessions of TMS per week separated by a minimum of $24 \mathrm{~h}$. The order of the sessions was counterbalanced across participants.

Tasks. All semantic judgments involved word-to-picture matching, with a probe picture presented alongside three words (i.e., a threealternative, forced-choice design). Figure 2 contains an example trial for each task. The thematic association task involved linking together things that were found or used together but did not have strongly overlapping physical features (e.g., the probe and target were not from the same semantic category). Relatively little executive control over semantic retrieval is thought to be required when the probe-target relationship is 
strong (e.g., a picture of an Alsatian presented with the word bone) since activation spreads rapidly and automatically between strongly related concepts. In contrast, in harder thematic trials when the probe-target relationship is weaker (e.g., a picture of an Alsatian presented with the words razor wire), participants may need to identify a linking context in which the probe and target can relate to each other, and then tailor semantic retrieval toward features relevant for this context (e.g., fierce) and away from dominant but currently irrelevant associations (e.g., dog as pet). A group of nine participants who did not take part in the repetitive TMS experiment rated the probe-target pairs for the strength of association on a 7 point scale $(0=$ no discernible association; $7=$ extremely strong link), and these ratings were used to assign trials to the strong and weak association conditions used in Analysis 1. Mean association strength for the strong association condition was 5.46 on a 7 point scale, while the mean association strength for weak associations was 4.70. We also supplemented this categorical comparison of strong- and weak-association trials in Analysis 1 with a parametric analysis of associative strength (Analysis 2; details below). The contrast of weak versus strong associations has been used in many investigations of semantic control: it reliably activates pMTG along with LIFG in fMRI studies (Badre et al., 2005; Noonan et al., 2013), and TMS to both LIFG and pMTG disrupts the retrieval of weak but not strong semantic associations (Whitney et al., 2011b), suggesting they both play a causal role in shaping semantic retrieval. Given these findings, we predicted that the disruptive effect of TMS would be inversely related to the strength of association between the probe and target concepts for pMTG but not AG.

In the identity-matching task, participants selected a target name for each probe picture. These judgments did not require participants to retrieve information about the context in which the probe objects were found or used; thus, if AG and/or pMTG specifically support thematic knowledge, this task should not show an effect of TMS (at least not above and beyond any nonspecific effects of stimulation). We varied the level of specificity at which items were identified, as follows: participants had to match a photograph of an object (e.g., a specific type of dog) to its superordinate label (animal) or to a more specific term (i.e., Alsatian). Specific-level matching requires similar representations with overlapping features to be distinguished (e.g., separation of the Alsatian from other breeds of dog). This task is impaired at an early stage in semantic dementia patients with ATL atrophy, as the distinguishing features of objects are lost (McClelland and Rogers, 2003; Rogers and McClelland, 2004; Patterson et al., 2007). As a result, we envisage that the stimulation of sites involved in the representation or retrieval of detailed conceptual information would likely disrupt specific-level matching more than superordinate-level matching. Identity-matching tasks also require some control over conceptual retrieval, although this might influence specific and superordinate trials in different ways. Specific trials require targets to be selected from a set of similar concepts, eliciting competition that might be stronger than that in superordinate trials (Rogers et al., 2015). However, superordinate trials involve a weaker match between the features in the picture and those activated by the target word (i.e., animal); therefore, semantic retrieval to superordinate labels might also require "shaping" to suit the demands of the task. As a consequence, we might expect stimulation to pMTG to disrupt both superordinate and specific matching if this region makes a critical contribution to different facets of semantic control, as suggested by Noonan et al. (2013).
Given the proximity of pMTG and AG to brain regions supporting aspects of visual processing and attention, we included a nonsemantic control task with perceptual and decision-making demands that are similar to the semantic judgments. The stimuli were pixelated and scrambled black-and-white photographs (initially of faces; Krieger-Redwood, 2012). Participants were asked to find targets that were identical to the probe; the distracters were the same images rotated by $180^{\circ}$ or $270^{\circ}$.

Task difficulty. Since our focus is on a functional dissociation between AG and pMTG, task difficulty is not able explain the full pattern of TMS results across sites. However, tasks requiring more controlled retrieval should have longer response times (RTs) and potentially lower accuracy than more automatic semantic judgments. In line with these predictions, response efficiency in baseline sessions for all sites (i.e., RT divided by the proportion of trials correct) was poorer for weak than strong thematic associations in the absence of TMS (Table 1; efficiency for strong associations $=1361.2$; efficiency for weak associations $=1845.3 ; t_{(20)}=17.51$, $p \leq 0.001)$. Thematic association judgments were also more difficult than trials involving identity matching $\left(t_{(41)}=10.41, p \leq 0.001\right)$. Within the identity-matching task, superordinate trials (mean efficiency $=$ 1158.7) were somewhat easier than specific trials overall (mean efficiency $=1203.0 ; t_{(20)}=2.34, p=0.030$ ). However, as can be seen in Table 1, specific judgments tended to be faster yet less accurate than superordinate ones.

Participants. Eighteen right-handed participants were recruited from the University of York (York, UK; seven females; mean age, 23 years; age range, 11 years). Three additional participants with incomplete data were removed before analysis. All participants had normal or corrected-tonormal vision and met safety screening requirements for TMS. Ethical 


\section{Identity matching}

Superordinate

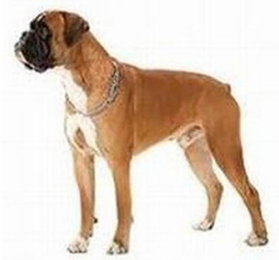

Animal

Tool
Plant

\begin{abstract}
Bulldog
\end{abstract}

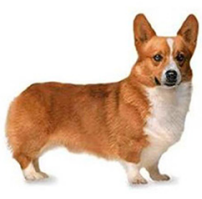

Specific

\section{Thematic matching}

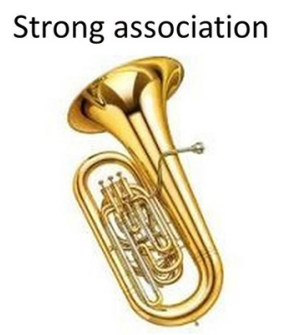

Zoo

Orchestra Driver

\section{Cake Hospital Pirate}

Figure 2. Examples of the identity judgments at different levels of specificity, and with thematic judgments tapping strong and weak associations. For both tasks, target words are underlined.

approval was given by the Ethics Committee of the York Neuroimaging Centre.

Stimuli. Three hundred eighteen color photographs, collected through an internet search engine (Google), were used in the semantic tasks (with the same probes used across the different judgment types). All images were resized to $200 \times 200$ pixels while maintaining the aspect ratio of the initial image to prevent distortions. Two hundred sixty-two images of faces were pixelated and scrambled for the visual control task (KriegerRedwood, 2012). In the semantic tasks, these images were presented alongside target nouns, which were either the names of natural/manmade objects at different levels of specificity or concrete associates of the object. The distracters in the thematic matching task were associates taken from other trials (carefully selected to ensure that they were not linked to the probe).

For the thematic association trials, we gathered ratings of association strength between the target and probe from 28 participants and these were used as a continuous variable in a parametric analysis of reaction time as a function of association strength and TMS (see Analysis 2). In addition, a set of nine participants rated all of the target concepts for familiarity on a 7 point scale. For these targets, we also obtained counts of lexical frequency using the SUBTLEX-UK database (van Heuven et al., 2014) and polysemy using WordNet (Fellbaum, 1998). Thus, in Analysis 2 , we were able to statistically control for the familiarity of the target concept, plus the lexical frequency of the words used to denote this concept, and the variability of the meaning of the target concept. In Analysis 3 of the identity-matching task, the same superordinate target words were repeated many times across trials. Therefore, in this analysis, we statistically controlled for conceptual variables relating to the probe as opposed to psycholinguistic variables related to the target. We collected typicality ratings for each probe concept from 15 participants, who were asked to judge on a 7 point scale whether each item (denoted by its specific verbal label) was a good example of its superordinate category using the methods of Rosch (1975). We also collected familiarity ratings, on a 7 point scale, for each probe concept from nine participants. In all cases, the participants providing these ratings did not take part in the TMS experiment.
Procedure. Structural T-1 MRI scans (3D FSPGR MRI) were coregistered to the scalp using Brainsight (Rogue Research, https:// www.rogue-research.com) to identify the stimulation targets in each participant's brain. Scalp measurements were made to determine the location of the vertex. At the beginning of each session, each participant's active motor threshold was established, determined as the lowest stimulation intensity needed to cause a contraction of the first dorsal interosseous muscle of the right hand when stimulating left motor cortex (average motor threshold, 61\%; motor threshold range, $29 \%$; average stimulator output, $73 \%$ ). TMS stimulation was delivered using an $8 \mathrm{~mm}$ figure-of-eight coil, attached to a MagStim Rapid stimulator (The Magstim Company Ltd). Repetitive trains of TMS were delivered at $1 \mathrm{~Hz}$ for $10 \mathrm{~min}$, changing the coil after $5 \mathrm{~min}$. Stimulation intensity was set at $120 \%$ of a participant's individual motor threshold and capped at a maximum of $75 \%$ of stimulator output. Coil orientation was adjusted to produce the most comfortable stimulation for participants.

Tasks were presented on a Windows PC running E-prime 2.0 (Psychology Software Tools). Each session started with a set of 20 practice trials. Per session, there were four blocks of trials after TMS and at baseline (i.e., in the absence of TMS). The blocks were divided into miniblocks containing 20 trials for each of the identity-matching and thematic matching conditions (with these split into 10 high-association/ low-association thematic trials and 10 superordinate/specific trials), and 10 trials for the visual control task (i.e., 50 trials in all per block; 40 trials per condition across blocks). The order of conditions and stimuli was counterbalanced across participants and experimental sessions. No trials were repeated within a session, and $7.5 \%$ of trials were repeated across sessions to ensure that there were equal numbers of trials per TMS session. Repeated trials were coded as dummy trials and removed before analysis. Tasks started with a prompt to remind the participant what feature they had to match (identity = match the picture with its name; association $=$ match the picture to a thematically associated item; visual $=$ match the top picture to an identical but rotated target picture). For all tasks, the three response options appeared $900 \mathrm{~ms}$ before the probe image, which stayed on screen for a maximum of $3000 \mathrm{~ms}$ after the presentation of the response image or until the participant made their response. Participants pressed 1,2, or 3 to indicate their selection of targets on the left, middle, and bottom of the screen.

Statistical analysis. Behavioral data were analyzed using hierarchical linear modeling in SAS version 9.3 (SAS Institute). Accuracy data were analyzed using PROC GLIMIX, but this analysis did not reveal significant effects of TMS and is not discussed further. Thus, the analyses below focus on RT, which was analyzed using PROC MIXED with maximum likelihood as the estimation method and an "unstructured" variancecovariance structure specified for the random effects. Incorrect trials were removed before analysis, as were outlying responses that fell $>2$ SDs from each participant's mean for that condition.

\section{Results}

\section{Analysis 1: omnibus model}

This analysis examined the effects of TMS for each site and condition. It included the following three categorical fixed effects: (1) condition, with five levels, superordinate identity matching (for the overarching category labels animal, weapon, food, vehicle, plant, clothes, household item, footwear, tree, instrument, and sports equipment), specific identity matching (using the names of individual objects), thematic association matching for strong probe-target pairings, thematic association matching for weaker probe-target pairings, and visual matching for meaningless images; (2) stimulation site with the four levels vertex, AG, pMTG, and ATL (although no significant behavioral disruption was observed following TMS to ATL, the data were included in the omnibus model to optimize estimates of RT in each condition in the absence of TMS); and (3) TMS with two levels, baseline and post-TMS. We fitted a fully saturated model in which random variation for the intercept was allowed for subject, target, and 


\begin{tabular}{|c|c|c|c|c|c|c|c|c|}
\hline \multirow[b]{3}{*}{ Measure } & \multirow[b]{3}{*}{ Condition } & \multirow[b]{3}{*}{ Baseline/TMS } & \multicolumn{6}{|l|}{ Site } \\
\hline & & & \multicolumn{2}{|l|}{$A G$} & \multicolumn{2}{|l|}{ pMTG } & \multicolumn{2}{|l|}{ Vertex } \\
\hline & & & Mean & SD & Mean & SD & Mean & SD \\
\hline \multirow[t]{10}{*}{ RT (ms) } & \multirow[t]{2}{*}{ Superordinate identity } & Baseline & 1108.4 & 347.5 & 1090.9 & 334.17 & 1112.3 & 370.92 \\
\hline & & TMS & 1123.4 & 359.16 & 1134.8 & 342.57 & 1103.8 & 356.55 \\
\hline & \multirow[t]{2}{*}{ Specific identity } & Baseline & 1073 & 384.49 & 1107.7 & 403.54 & 1104.3 & 393.47 \\
\hline & & TMS & 1129.1 & 429.15 & 1147.3 & 406.1 & 1143.7 & 420.72 \\
\hline & \multirow[t]{2}{*}{ Strong association } & Baseline & 1297.7 & 460.89 & 1312.4 & 447.95 & 1297.2 & 440.25 \\
\hline & & TMS & 1331.4 & 452.74 & 1138.7 & 447.18 & 1311.9 & 417.18 \\
\hline & \multirow{2}{*}{ Weak association } & Baseline & 1528.6 & 517.36 & 1582.9 & 546.06 & 1593.7 & 530.27 \\
\hline & & TMS & 1590.8 & 544.35 & 1616 & 537.65 & 1605.8 & 542.56 \\
\hline & \multirow[t]{2}{*}{ Visual } & Baseline & 1205.7 & 428.56 & 1189.8 & 423.17 & 1255.1 & 407.51 \\
\hline & & TMS & 1247.8 & 465.81 & 1249 & 421.25 & 1286.5 & 462.89 \\
\hline \multirow[t]{10}{*}{ Accuracy (proportion correct) } & \multirow[t]{2}{*}{ Superordinate identity } & Baseline & 0.96 & 0.052 & 0.97 & 0.025 & 0.97 & 0.033 \\
\hline & & TMS & 0.96 & 0.040 & 0.97 & 0.033 & 0.97 & 0.031 \\
\hline & \multirow[t]{2}{*}{ Specific identity } & Baseline & 0.92 & 0.070 & 0.93 & 0.049 & 0.93 & 0.044 \\
\hline & & TMS & 0.92 & 0.057 & 0.91 & 0.051 & 0.92 & 0.440 \\
\hline & \multirow[t]{2}{*}{ Strong association } & Baseline & 0.96 & 0.033 & 0.95 & 0.035 & 0.97 & 0.035 \\
\hline & & TMS & 0.96 & 0.027 & 0.97 & 0.034 & 0.96 & 0.049 \\
\hline & \multirow[t]{2}{*}{ Weak association } & Baseline & 0.91 & 0.072 & 0.89 & 0.064 & 0.89 & 0.087 \\
\hline & & TMS & 0.92 & 0.056 & 0.89 & 0.091 & 0.89 & 0.071 \\
\hline & \multirow[t]{2}{*}{ Visual } & Baseline & 0.93 & 0.063 & 0.92 & 0.076 & 0.90 & 0.100 \\
\hline & & TMS & 0.93 & 0.076 & 0.93 & 0.050 & 0.92 & 0.080 \\
\hline
\end{tabular}

Table 2. Paired contrasts examining the TMS effect (no TMS vs TMS) for each site and each task separately (Analysis 1)

\begin{tabular}{lllll}
\hline Site & $\begin{array}{l}\text { LS mean estimate } \\
\text { difference }\end{array}$ & Condition & $t$ Value & Significance \\
\hline AG & -18.81 & Superordinate identity & -0.92 & 0.357 \\
AG & -57.20 & Specific identity & $-\mathbf{2 . 7 1}$ & $\mathbf{0 . 0 0 7}$ \\
AG & -43.80 & Strong association & $-\mathbf{2 . 1 3}$ & $\mathbf{0 . 0 3 4}$ \\
AG & -64.28 & Weak association & $-\mathbf{3 . 0 0}$ & $\mathbf{0 . 0 0 3}$ \\
AG & -41.50 & Visual & $-\mathbf{1 . 9 9}$ & $\mathbf{0 . 0 4 6}$ \\
pMTG & -43.20 & Superordinate identity & $\mathbf{- 2 . 1 1}$ & $\mathbf{0 . 0 3 5}$ \\
pMTG & -46.06 & Specific identity & $-\mathbf{2 . 1 9}$ & $\mathbf{0 . 0 2 8}$ \\
pMTG & -20.20 & Strong association & -0.98 & 0.326 \\
pMTG & -38.30 & Weak association & -1.76 & $\mathbf{0 . 0 7 9}$ \\
pMTG & -53.29 & Visual & $\mathbf{- 2 . 5 4}$ & $\mathbf{0 . 0 1 1}$ \\
\hline
\end{tabular}

Comparing LS means for each site and task, with and without TMS. Significant effects of TMS are highlighted in bold text.

probe. This allowed us to estimate pairwise comparisons of the least squared (LS) mean reaction times, with and without TMS, controlling for multiple comparisons, separately at each site for each condition (a total of 26,001 observations).

Table 1 shows the mean RT and the accuracy for each condition for each site, as a function of TMS. Table 2 shows the outcomes of the multiple pairwise comparisons derived from the omnibus model. As expected, we found no significant TMS effects at vertex (the nonsemantic control site). AG showed significant TMS effects for specific identity matching, strong and weak thematic associations, and visual decisions. pMTG showed significant TMS effects for superordinate and specific identity matching, and visual decisions, and a marginal effect for weak thematic associations. Since TMS to both AG and pMTG disrupted identity and thematic association matching, neither site showed the profile expected for a thematic hub (i.e., specific disruption of semantic associations). However, since stimulation to these sites disrupted visual-matching as well as semantic decisions, it might be that neighboring cortical regions were also influenced by the stimulation or that the function of pMTG/AG is not restricted to the semantic domain but extends to visual and/or executive aspects of cognition (a view that is compatible with the existing literature; for comparison, see Humphreys and Lambon Ralph, 2014). For this reason, we included the TMS effect for the visual task (per participant and session) as a covariate of no interest in subsequent analyses examining association strength and specificity.

\section{Analysis 2: association strength as a continuous variable}

This analysis examined the effects of associative strength for the thematic matching task in more detail. We fitted a fully saturated model to estimate the fixed effects of TMS (stimulation vs no stimulation), site (pMTG, AG), and associative strength (modeled as a continuous variable) on RT, with subject and target as random effects. This model was computed twice: first, statistically controlling for target lexical frequency, polysemy, and rated conceptual familiarity (Analysis 2a; 3648 observations); and, second, controlling for the effects of TMS on the visual task, by entering the LS means estimates of this nonsemantic TMS effect, per participant and per site, as a covariate (Analysis 2b; 4773 observations). We predicted that TMS to pMTG would only disrupt the weakest association trials, while TMS to AG might disrupt stronger thematic associations.

Figure 3 shows the predicted RT values at different levels of associative strength while controlling for all other sources of variance in the model (Analysis 2a). Point comparisons were computed for the LS means with and without TMS at six different levels of associative strength (ranging from 1.5 to 6.5 on a 7 point scale). These comparisons, corrected for multiple comparisons, confirmed our predictions. Stimulation to pMTG caused a specific disruption for weakly associated items, while stimulation to AG disrupted strong-association trials. These effects were observed at both sites even when the disruption to the visual trials was entered as a covariate (Analysis 2b; i.e., the disruption of nonsemantic decisions was not sufficient to explain the impairment of semantic performance; Table 3). This dissociation between pMTG and AG in the thematic association-matching task is consistent with their hypothesized role in more controlled and automatic aspects of semantic retrieval, respectively. 
AG
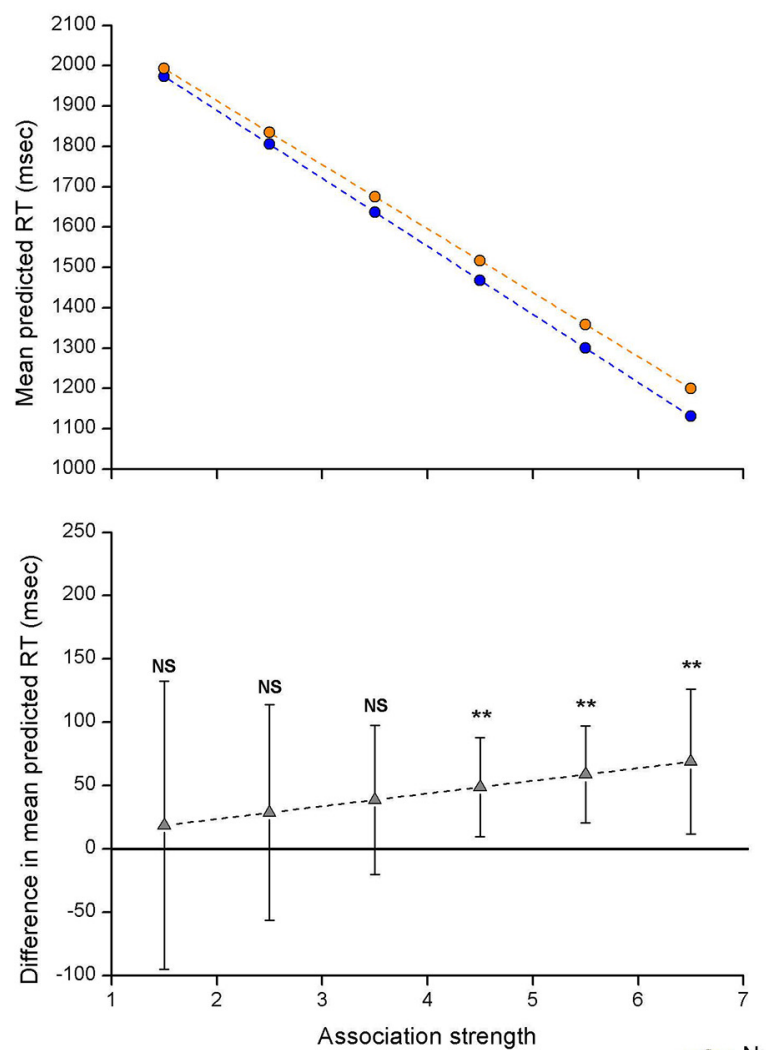

-- - No TMS

$--\mathrm{O}-\mathrm{TMS}$

-- $\triangle$ - No TMS/TMS difference
PMTG
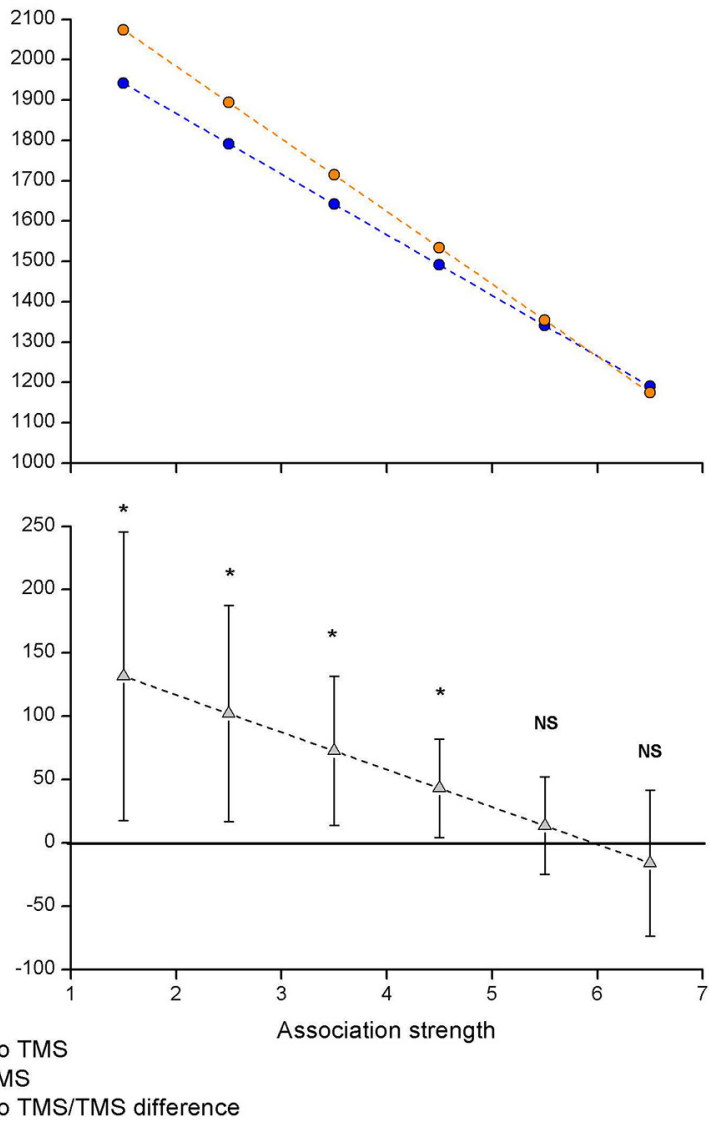

Figure 3. Predicted RT values for AG and pMTG in Analysis 2a, modeling association strength as a continuous variable. The top plots show the predicted RT with and without TMS at different levels of associative strength, while the bottom plots show the difference between these conditions. Error bars represent $95 \%$ confidence intervals. NS, No significant effect of $\mathrm{TMS}^{*} p<0.05 ;{ }^{* *} p<0.01$.

Table 3. Paired contrasts of the TMS effect (no TMS vs TMS) in Analysis 2, modeling associative strength as a continuous variable

\begin{tabular}{|c|c|c|c|c|c|}
\hline \multirow[b]{2}{*}{ Site } & \multirow{2}{*}{$\begin{array}{l}\text { Associative } \\
\text { strength }\end{array}$} & \multicolumn{2}{|c|}{$\begin{array}{l}\text { No visual covariate } \\
\text { (Analysis 2a) }\end{array}$} & \multicolumn{2}{|c|}{$\begin{array}{l}\text { Visual covariate entered } \\
\text { (Analysis 2b) }\end{array}$} \\
\hline & & $t$ & Significance & $t$ & Significance \\
\hline \multirow[t]{6}{*}{ AG } & 1.5 & -0.32 & 0.747 & -0.86 & 0.388 \\
\hline & 2.5 & -0.66 & 0.508 & -1.12 & 0.261 \\
\hline & 3.5 & -1.30 & 0.195 & -1.58 & 0.113 \\
\hline & 4.5 & -2.46 & 0.014 & -2.31 & 0.021 \\
\hline & 5.5 & -3.02 & 0.003 & -2.25 & 0.025 \\
\hline & 6.5 & -2.36 & 0.018 & -1.45 & 0.146 \\
\hline \multirow[t]{6}{*}{ pMTG } & 1.5 & -2.26 & 0.024 & -1.98 & 0.048 \\
\hline & 2.5 & -2.35 & 0.019 & -2.03 & 0.042 \\
\hline & 3.5 & -2.42 & 0.016 & -2.07 & 0.039 \\
\hline & 4.5 & -2.17 & 0.030 & -1.77 & 0.077 \\
\hline & 5.5 & -0.70 & 0.485 & -0.45 & 0.653 \\
\hline & 6.5 & 0.54 & 0.591 & 0.59 & 0.556 \\
\hline
\end{tabular}

Point comparisons of RT with and without TMS at different levels of associativestrength. The analysis was performed twice, with and without the inclusion of the TMS effect for the visual task as a covariate. Significant effects of TMS at $p<0.05$ are highlighted in bold text.

Analysis 3: identity matching at two levels of specificity This analysis examined the effect of TMS on identity matching in more detail. We fitted a fully saturated model to estimate the fixed effects of TMS (stimulation vs no stimulation), site (pMTG, AG), and specificity (superordinate vs specific), with subject and target as random effects. This model was computed twice: first, statistically controlling for concept typical- ity and familiarity (Analysis 3a; 4368 observations); and, second, controlling for the effects of TMS on the visual task by entering the LS means estimates of this nonsemantic TMS effect, per participant and per site, as a covariate (Analysis 3b; 5091 observations).

Figure 4 shows the predicted RT values for superordinate and specific identity matching while controlling for all other sources of variance in the model (Analysis 3a). Paired contrasts, corrected for multiple comparisons (Fig. 4, Table 4), revealed that TMS to AG disrupted identity matching at the specific but not at the superordinate level, suggesting that this site plays a necessary role in precise semantic retrieval that differentiates highly similar concepts that share many features. In contrast, stimulation of pMTG elicited significant disruption for both the specific and superordinate trials, demonstrating a functional dissociation for these two sites on a second task. A similar pattern was seen when the visual TMS effect for each participant and each site was added as a covariate of no interest (Analysis 3b). Thus, AG and pMTG appear to contribute to aspects of semantic cognition beyond the storage and/or retrieval of thematic associations, even when general effects on nonsemantic visual processing are taken into account.

\section{Discussion}

This study examined the following two contrasting hypotheses about the role of AG and pMTG: (1) by one view, these sites store semantic information about thematic associations and events but are not critical for identity matching (compare with 
AG
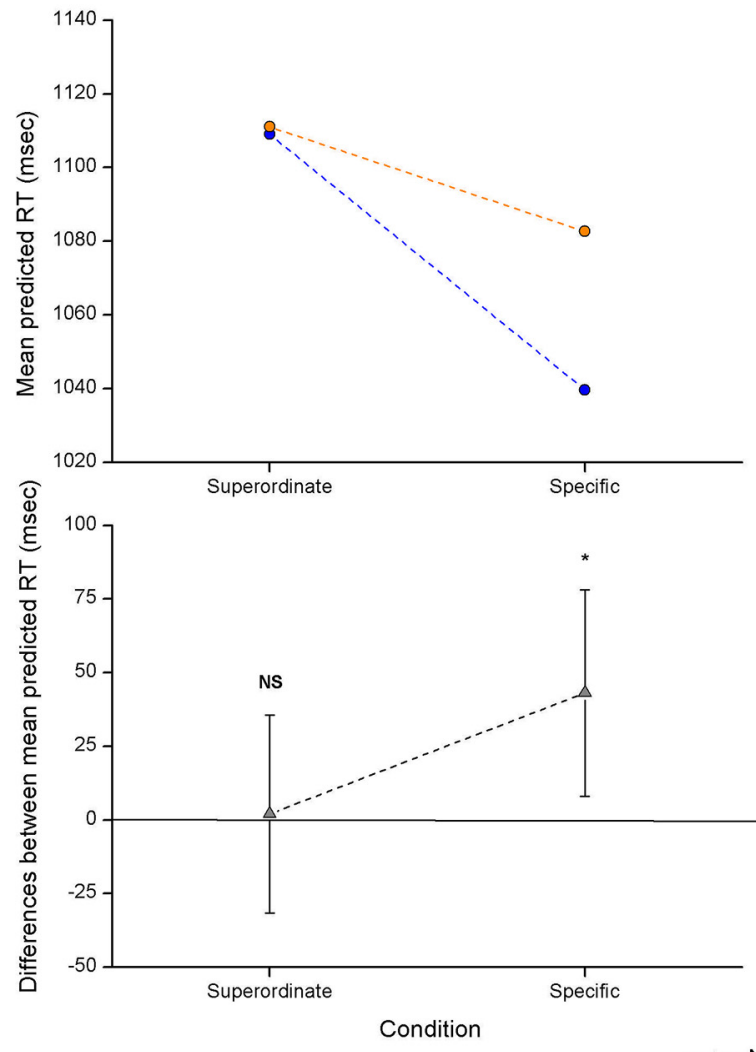

PMTG
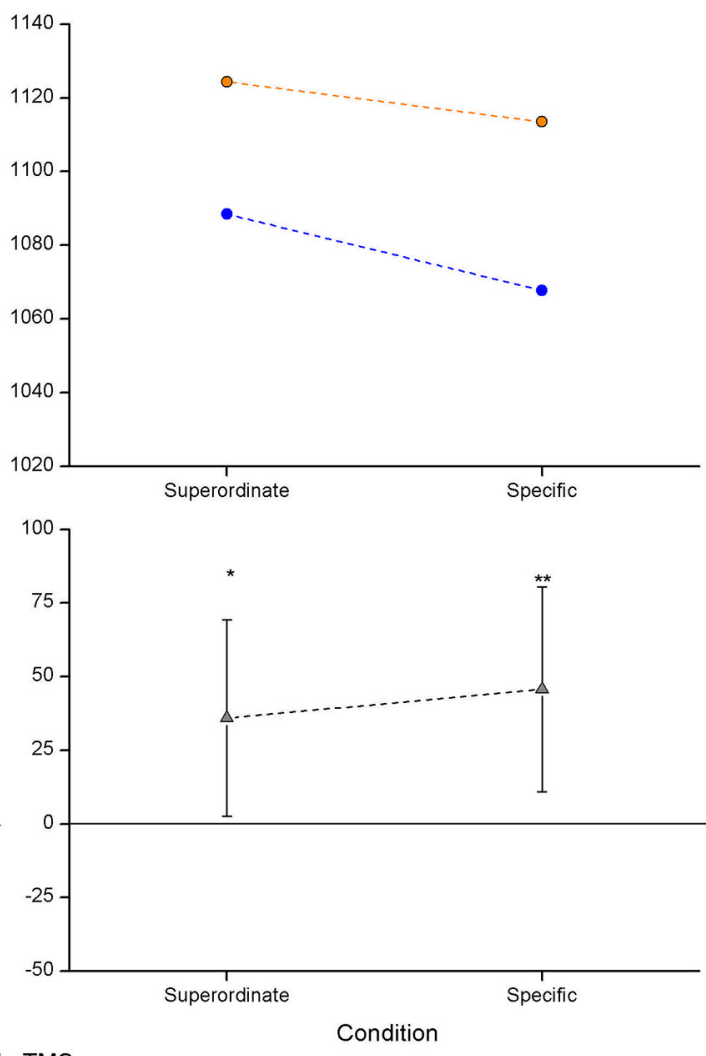

No TMS

--o- TMS

- $\triangle$ - No TMS/TMS difference

Figure 4. Predicted RT values for AG and pMTG in Analysis 3a, comparing identity matching at two levels of specificity. The top plots show the predicted RT with and without TMS at different levels of specificity, while the bottom plots show the difference between these conditions. Error bars represent $95 \%$ confidence intervals. NS, No significant effect of TMS; ${ }^{*} p<0.05 ;{ }^{* *} p<0.01$.

Table 4. Paired contrasts of TMS effect (no TMS vs TMS) in Analysis 3 at two levels of specificity

\begin{tabular}{|c|c|c|c|c|c|}
\hline \multirow[b]{2}{*}{ Site } & \multirow[b]{2}{*}{ Specificity } & \multicolumn{2}{|c|}{$\begin{array}{l}\text { No visual covariate } \\
\text { (Analysis 3a) }\end{array}$} & \multicolumn{2}{|c|}{$\begin{array}{l}\text { Visual covariate entered } \\
\text { (Analysis 3b) }\end{array}$} \\
\hline & & $t$ & Significance & $t$ & Significance \\
\hline \multirow[t]{2}{*}{ AG } & Superordinate & -0.12 & 0.906 & 0.62 & 0.538 \\
\hline & Specific & -2.41 & 0.016 & -1.76 & 0.079 \\
\hline \multirow[t]{2}{*}{ pMTG } & Superordinate & -2.11 & 0.035 & -1.26 & 0.208 \\
\hline & Specific & -2.58 & 0.010 & -1.72 & 0.085 \\
\hline
\end{tabular}

Comparisons of LS means with and without TMS for superordinate identity-matching trials (involving a category label such as "animal"), and specific-level trials. The analysis was performed twice, with and without the inclusion of the TMS effect for the visual task as a covariate. Significant effects of TMS are highlighted in bold text.

the "two hub theory"; Schwartz et al., 2011; Mirman and Graziano, 2012a,b); and (2) alternatively, these sites support controlled versus automatic aspects of semantic retrieval (Jefferies, 2013; Noonan et al., 2013). TMS was applied to peaks taken from two meta-analyses of semantic cognition examining (1) semantic over nonsemantic tasks matched for difficulty (Binder et al., 2009; peak in mid-AG); and (2) semantic decisions with high and low control demands (Noonan et al., 2013; peak in pMTG). We measured the effects of this stimulation on identity matching (at superordinate and specific levels) and thematic matching (for weak and strong associations). The results show that AG and pMTG make dissociable contributions to semantic cognition, which is consistent with a theoretical model in which pMTG (alongside LIFG) underpins controlled aspects of semantic retrieval. In contrast, AG (alongside ATL) supports more automatic processes that are critical to the retrieval of specific concepts.

Without any control over the retrieval of knowledge, semantic processing is dominated by spreading activation between strongly linked concepts, yet we show a high degree of flexibility in the information we retrieve-we can selectively focus on nondominant aspects of meaning when required to do so by a task. Coactivation of pMTG and LIFG has been linked to this type of controlled semantic retrieval (Jefferies, 2013; Noonan et al., 2013). For example, when asked to recover an unusual thematic association between weakly associated concepts (e.g., Alsatian and razor wire), dominant features/associations are largely irrelevant (i.e., "pet"), yet we can bring these disparate concepts together by focusing on features that are relevant to establishing a connection (e.g., "fierce"). Studies examining neural responses to weak over strong semantic associations, and ambiguous over unambiguous words, implicate pMTG and LIFG in this type of controlled shaping of semantic retrieval (Badre et al., 2005; Whitney et al., 2011 a,b; Noonan et al., 2013). Moreover, damage to either LIFG or posterior temporal areas, including pMTG in patients with SA, is associated with deregulated semantic retrieval that is characterized by a tendency to retrieve dominant features and associations, even when these are irrelevant to the current task or context (Jefferies and Lambon Ralph, 2006; Noonan et al., 2010). Thus, our finding that the stimulation of pMTG disrupted judgments about weak but not strong thematic associations adds 
anatomical specificity to neuropsychological findings and demonstrates causality.

We also found that stimulation of pMTG disrupted identity matching for both specific and superordinate trials. This result is not unexpected given that these judgments also involved controlled retrieval. Specific identity matching requires selection of a target concept from among highly similar items; therefore, control processes might have been engaged to overcome competition. Superordinate identity matching involves terms like "animal" that have variable meanings across contexts. For these items, automatic spreading activation is not expected to strongly overlap with the features visible in the probe picture, and thus control processes may be engaged to tailor retrieval from the words to suit the demands of the task. Consistent with this account, SA patients have deficits on both identity-matching and thematic matching tasks that reflect the control requirements of these judgments (Noonan et al., 2010; Rogers et al., 2015). In summary, although pMTG activation continues to be interpreted in terms of a semantic store (Binder et al., 2009; Martin et al., 2014), multiple studies using complementary methods have noted responses incompatible with a role in passive storage and suggest instead that pMTG allows for the controlled retrieval of semantic activation (Gold et al., 2005; Whitney et al., 2011b; Jefferies, 2013; Noonan et al., 2013).

The mid-AG site demonstrated a different response profile from pMTG; TMS to AG elicited greater disruption of strongly related thematic associations, which is in line with the proposal that this site contributes to more automatic forms of retrieval. AG has several functional subdivisions (Seghier et al., 2010; Noonan et al., 2013), as follows: dorsal AG is associated with executive semantic processing, while the mid-AG region (our TMS target site) is typically characterized by task-related deactivation (Seghier et al., 2010; Wirth et al., 2011; Humphreys and Lambon Ralph, 2014; i.e., it forms part of the default-mode network). This deactivation is more pronounced for harder semantic and nonsemantic tasks (Binder et al., 2003; Sabsevitz et al., 2005; Humphreys et al., 2015), which is consistent with the view that this region does not facilitate executively demanding aspects of cognition. In contrast, it shows activation for tasks that involve the retrieval of internal representations, including automatic semantic tasks, episodic memory, numerical fact retrieval, and sentence comprehension (Humphreys and Lambon Ralph, 2014).

Our observation of greater disruption for specific than subordinate-level identity matching following AG stimulation might also be explicable within this framework. For example, mid-AG could contribute to the reflexive (automatic) allocation of attention to activated concepts. Consequently, when a picture of a corgi is presented alongside different dog names, attention might be captured by the features of corgi that are activated in the semantic store. If TMS to AG results in less accurate allocation of attention, this could reduce the ability to distinguish between similar representations (e.g., corgi and Dalmatian in specific trials), but the ability to separate dogs from human-made objects in superordinate trials would be preserved. This proposal is consistent with the broader role of posterior inferior parietal regions in reflexive attention to memory (Corbetta et al., 2008; Hutchinson et al., 2009). Although this account is compatible with the current data, further research is needed to confirm this interpretation.

TMS additionally disrupted a visual matching control task, and we statistically controlled for this effect in the analysis. Although our tasks and interpretation focus on the necessary contribution of mid-AG and pMTG to semantic cognition, the framework above does not preclude the possibility that these brain regions also contribute to nonsemantic processes, or lie adjacent to visual or executive regions that were influenced by stimulation in some of the participants. Although a recent metaanalysis examining semantic control found that PMTG made a relatively specific contribution to semantic decisions (relative to phonological decisions; Noonan et al., 2013), it lies anterior to the visual motion-processing area MT (Malach et al., 1995; Wallentin et al., 2011) and immediately above ventral occipital-temporal areas coupled to the frontoparietal control system (Duncan, 2010; Yeo et al., 2011). Similarly, AG is engaged in many nonsemantic tasks, and there is an anterior-to-posterior transition from stimulus-driven attention in supramarginal gyrus to semantics in AG (Humphreys and Lambon Ralph, 2014). TMSinduced disruption of these processes might account for effects on the visual-matching task.

The current study focused on the functional differentiation of pMTG and AG, yet, as noted in the Introduction, these sites are similarly recruited by "event semantics" and "thematic judgments" (Schwartz et al., 2011; de Zubicaray et al., 2013). The two-hub account proposes that one or both of these sites represent thematic associations as opposed to the concrete features of objects. This proposal was originally motivated by the observation that patients with temporoparietal stroke make thematic errors in picture naming (e.g., "squirrel" $\rightarrow$ "nuts"), unlike those with ATL damage (Jefferies and Lambon Ralph, 2006; Schwartz et al., 2011). However, this inappropriate production of associations in picture naming might reflect intact thematic knowledge but a deficit in the ability to constrain semantic retrieval, which is in line with these patients' deficits in semantic control (Jefferies and Lambon Ralph, 2006; Noonan et al., 2010). The current findings are largely inconsistent with the predictions of the two-hub account since TMS to both sites disrupted identity as well as thematic matching, even after the nonspecific effects of TMS to these sites was taken into account. Given that events can have very different meanings in different contexts (e.g., kicking ball: on a field $=$ game vs in a greenhouse $=$ vandalism), the contribution of pMTG to action/event understanding might follow from its role in shaping retrieval in line with the current context. In contrast, AG might play a critical role in the orientation of attention toward specific event representations and/or maintain a focus of attention across a sequence of interacting concepts (Humphreys and Lambon Ralph, 2014).

To conclude, although semantic contrasts can activate pMTG and mid-AG as a single cluster (Binder et al., 2009), we propose that these sites should not be interpreted narrowly in terms of supporting specific aspects of semantic knowledge-instead, they are implicated within distinct large-scale cortical networks, which support retrieval processes crucial for semantics and also other aspects of cognition. Our two stimulation sites have different functional connectivity profiles revealed by resting-state fMRI (Fig. 1); mid-AG has strong connections to ATL (Ruschel et al., 2014) and to posterior cingulate and ventromedial prefrontal cortex implicated in the default mode network (Uddin et al., 2010). In contrast, pMTG shows strong connectivity to LIFG, implicated in semantic control (Koopmans et al., 2012). These different patterns of connectivity support our conclusions that mid-AG is implicated in automatic aspects of retrieval, while pMTG contributes to controlled retrieval.

\section{References}

Ashburner J, Friston KJ (2005) Unified segmentation. Neuroimage 26:839851. CrossRef Medline

Badre D, Poldrack RA, Paré-Blagoev EJ, Insler RZ, Wagner AD (2005) Dis- 
sociable controlled retrieval and generalized selection mechanisms in ventrolateral prefrontal cortex. Neuron 47:907-918. CrossRef Medline

Bedny M, McGill M, Thompson-Schill SL (2008) Semantic adaptation and competition during word comprehension. Cereb Cortex 18:2574-2585. CrossRef Medline

Binder JR, McKiernan KA, Parsons ME, Westbury CF, Possing ET, Kaufman JN, Buchanan L (2003) Neural correlates of lexical access during visual word recognition. J Cogn Neurosci 15:372-393. CrossRef Medline

Binder JR, Desai RH, Graves WW, Conant LL (2009) Where is the semantic system? A critical review and meta-analysis of 120 functional neuroimaging studies. Cereb Cortex 19:2767-2796. CrossRef Medline

Cabeza R, Mazuz YS, Stokes J, Kragel JE, Woldorff MG, Ciaramelli E, Olson IR, Moscovitch M (2011) Overlapping parietal activity in memory and perception: evidence for the attention to memory model. J Cogn Neurosci 23:3209-3217. CrossRef Medline

Chao-Gan Y, Yu-Feng Z (2010) DPARSF: A MATLAB toolbox for "pipeline" data analysis of resting-state fMRI. Front Syst Neurosci 4:13. CrossRef Medline

Corbetta M, Patel G, Shulman GL (2008) The reorienting system of the human brain: from environment to theory of mind. Neuron 58:306-324. CrossRef Medline

de Zubicaray GI, Hansen S, McMahon KL (2013) Differential processing of thematic and categorical conceptual relations in spoken word production. J Exp Psychol Gen 142:131-142. CrossRef Medline

Duncan J (2010) The multiple-demand (MD) system of the primate brain: mental programs for intelligent behaviour. Trends Cogn Sci 14:172-179. CrossRef Medline

Fellbaum C (1998) WordNet. New York: Wiley Online Library.

Gold BT, Balota DA, Kirchhoff BA, Buckner RL (2005) Common and dissociable activation patterns associated with controlled semantic and phonological processing: evidence from FMRI adaptation. Cereb Cortex 15: 1438-1450. CrossRef Medline

Humphreys GF, Lambon Ralph MA (2014) Fusion and fission of cognitive functions in the human parietal cortex. Cereb Cortex. Advance online publication. Retrieved 14 September 2015. doi:10.1093/cercor/bhu198. CrossRef Medline

Humphreys GF, Hoffman P, Visser M, Binney RJ, Lambon Ralph MA (2015) Establishing task- and modality-dependent dissociations between the semantic and default mode networks. Proc Natl Acad Sci U S A 112:78577862. CrossRef Medline

Hutchinson JB, Uncapher MR, Wagner AD (2009) Posterior parietal cortex and episodic retrieval: convergent and divergent effects of attention and memory. Learn Mem 16:343-356. Medline

Jefferies E (2013) The neural basis of semantic cognition: converging evidence from neuropsychology, neuroimaging and TMS. Cortex 49:611625. CrossRef Medline

Jefferies E, Lambon Ralph MA (2006) Semantic impairment in stroke aphasia versus semantic dementia: a case-series comparison. Brain 129:21322147. CrossRef Medline

Koopmans PJ, Boyacioğlu R, Barth M, Norris DG (2012) Whole brain, high resolution spin-echo resting state fMRI using PINS multiplexing at $7 \mathrm{~T}$. Neuroimage 62:1939-1946. CrossRef Medline

Krieger-Redwood K (2012) An investigation of phonological and semantic control using TMS and fMRI. In: Psychology. York, UK: University of York.

Malach R, Reppas JB, Benson RR, Kwong KK, Jiang H, Kennedy WA, Ledden PJ, Brady TJ, Rosen BR, Tootell RB (1995) Object-related activity revealed by functional magnetic resonance imaging in human occipital cortex. Proc Natl Acad Sci U S A 92:8135-8139. CrossRef Medline

Martin A (2007) The representation of object concepts in the brain. Annu Rev Psychol 58:25-45. CrossRef Medline

Martin A, Simmons KW, Beauchamp MS, Gotts SJ (2014) Is a single "hub," with lots of spokes, an accurate description of the neural architecture of action semantics? Comment on "Action semantics: a unifying conceptual framework for the selective use of multimodal and modality-specific object knowledge" by van Elk, Van Schie and Bekkering. Physics Life Rev 11:261-262. CrossRef Medline

McClelland JL, Rogers TT (2003) The parallel distributed processing approach to semantic cognition. Nat Rev Neurosci 4:310-322. CrossRef Medline

Mirman D, Graziano KM (2012a) Damage to temporo-parietal cortex de- creases incidental activation of thematic relations during spoken word comprehension. Neuropsychologia 50:1990-1997. CrossRef Medline

Mirman D, Graziano KM (2012b) Indivdual differences in the strength of taxonomic versus thematic relations. J Exp Psychol Gen 141:601-609. CrossRef Medline

Noonan KA, Jefferies E, Corbett F, Lambon Ralph MA (2010) Elucidating the nature of deregulated semantic cognition in semantic aphasia: evidence for the roles of prefrontal and temporo-parietal cortices. J Cogn Neurosci 22:1597-1613. CrossRef Medline

Noonan KA, Jefferies E, Visser M, Lambon Ralph MA (2013) Going beyond inferior prefrontal involvement in semantic control: evidence for the additional contribution of dorsal angular gyrus and posterior middle temporal cortex. J Cogn Neurosci 25:1824-1850. CrossRef Medline

Nooner KB, Colcombe SJ, Tobe RH, Mennes M, Benedict MM, Moreno AL, Panek LJ, Brown S, Zavitz ST, Li Q, Sikka S, Gutman D, Bangaru S, Schlachter RT, Kamiel SM, Anwar AR, Hinz CM, Kaplan MS, Rachlin AB, Adelsberg S, et al. (2012) The NKI-Rockland sample: a model for accelerating the pace of discovery science in psychiatry. Front Neurosci 6:152. CrossRef Medline

Patterson K, Nestor PJ, Rogers TT (2007) Where do you know what you know? The representation of semantic knowledge in the human brain. Nat Rev Neurosci 8:976-987. CrossRef Medline

Pobric G, Jefferies E, Lambon Ralph MA (2010) Category-specific versus category-general semantic impairment induced by transcranial magnetic stimulation. Curr Biol 20:964-968. CrossRef Medline

Rodd JM, Johnsrude IS, Davis MH (2012) Dissociating frontotemporal contributions to semantic ambiguity resolution in spoken sentences. Cereb Cortex 22:1761-1773. CrossRef Medline

Rogers TT, McClelland JL (2004) Semantic cognition: a parallel distributed processing approach. Cambridge, MA: MIT.

Rogers TT, Lambon Ralph MA, Garrard P, Bozeat S, McClelland JL, Hodges JR, Patterson K (2004) Structure and deterioration of semantic memory: a neuropsychological and computational investigation. Psychol Rev 111:205-235. CrossRef Medline

Rogers TT, Patterson K, Jefferies E, Lambon Ralph MA (2015) Disorders of representation and control in semantic cognition: effects of familiarity, typicality, and specificity. Neuropsychologia. Advance online publication. Retrieved 14 September 2015. doi:10.1016/j.neuropsychologia.2015.04.015. CrossRef Medline

Rosch E (1975) Cognitive representations of semantic categories. J Exp Psychol Gen 104:192-233. CrossRef

Rosner BA (2006) Fundamentals of biostatistics, Ed 6. Belmont, CA: Thompson-Brooks/Cole.

Ruschel M, Knösche TR, Friederici AD, Turner R, Geyer S, Anwander A (2014) Connectivity architecture and subdivision of the human inferior parietal cortex revealed by diffusion MRI. Cereb Cortex 24:2436-2448. CrossRef Medline

Sabsevitz DS, Medler DA, Seidenberg M, Binder JR (2005) Modulation of the semantic system by word imageability. Neuroimage 27:188-200. CrossRef Medline

Schwartz MF, Kimberg DY, Walker GM, Brecher A, Faseyitan OK, Dell GS, Mirman D, Coslett HB (2011) Neuroanatomical dissociation for taxonomic and thematic knowledge in the human brain. Proc Natl Acad Sci U S A 108:8520-8524. CrossRef Medline

Seghier ML, Fagan E, Price CJ (2010) Functional subdivisions in the left angular gyrus where the semantic system meets and diverges from the default network. J Neurosci 30:16809-16817. CrossRef Medline

Thompson-Schill SL, D’Esposito M, Aguirre GK, Farah MJ (1997) Role of left inferior prefrontal cortex in retrieval of semantic knowledge: a reevaluation. Proc Natl Acad Sci U S A 94:14792-14797. CrossRef Medline

Tyler LK, Stamatakis EA, Dick E, Bright P, Fletcher P, Moss H (2003) Objects and their actions: evidence for a neurally distributed semantic system. Neuroimage 18:542-557. CrossRef Medline

Uddin LQ, Supekar K, Amin H, Rykhlevskaia E, Nguyen DA, Greicius MD, Menon V (2010) Dissociable connectivity within human angular gyrus and intraparietal sulcus: evidence from functional and structural connectivity. Cereb Cortex 20:2636-2646. CrossRef Medline

van Heuven WJB, Mandera P, Keuleers E, Brysbaert M (2014) SUBTLEXUK: a new and improved word frequency database for British English. Q J Exp Psychol (Hove) 67:1176-1190. CrossRef Medline

Visser M, Jefferies E, Embleton KV, Lambon Ralph MA (2012) Both the 
middle temporal gyrus and the ventral anterior temporal area are crucial for multimodal semantic processing: distortion-corrected fMRI evidence for a double gradient of information convergence in the temporal lobes. J Cogn Neurosci 24:1766-1778. CrossRef Medline

Wagner AD, Maril A, Bjork RA, Schacter DL (2001) Prefrontal contributions to executive control: fMRI evidence for functional distinctions within lateral prefrontal cortex. Neuroimage 14:1337-1347. CrossRef Medline

Wallentin M, Nielsen AH, Vuust P, Dohn A, Roepstorff A, Lund TE (2011) BOLD response to motion verbs in left posterior middle temporal gyrus during story comprehension. Brain Lang 119:221-225. CrossRef Medline

Whitney C, Jefferies E, Kircher T (2011a) Heterogeneity of the left temporal lobe in semantic representation and control: priming multiple versus single meanings of ambiguous words. Cereb Cortex 21:831-844. CrossRef Medline

Whitney C, Kirk M, O'Sullivan J, Lambon Ralph MA, Jefferies E (2011b) The neural organization of semantic control: TMS evidence for a distrib- uted network in left inferior frontal and posterior middle temporal gyrus. Cereb Cortex 21:1066-1075. CrossRef Medline

Whitney C, Kirk M, O’Sullivan J, Lambon Ralph MA, Jefferies E (2012) Executive semantic processing is underpinned by a large-scale neural network: revealing the contribution of left prefrontal, posterior temporal, and parietal cortex to controlled retrieval and selection using TMS. J Cogn Neurosci 24:133-147. CrossRef Medline

Wirth M, Jann K, Dierks T, Federspiel A, Wiest R, Horn H (2011) Semantic memory involvement in the default mode network: a functional neuroimaging study using independent component analysis. Neuroimage 54: 3057-3066. CrossRef Medline

Yeo BT, Krienen FM, Sepulcre J, Sabuncu MR, Lashkari D, Hollinshead M, Roffman JL, Smoller JW, Zöllei L, Polimeni JR, Fischl B, Liu H, Buckner RL (2011) The organization of the human cerebral cortex estimated by intrinsic functional connectivity. J Neurophysiol 106:1125-1165. CrossRef Medline 